\title{
LOWER RADICALS IN NONASSOCIATIVE RINGS
}

\author{
R. TANGEMAN and D. KREILING
}

(Received 2nd April 1970)

Communicated by G. E. Wall

Let $W$ be a universal class of (not necessarily associative) rings and let $A \subseteq W$. Kurosh has given in [6] a construction for $L A$, the lower radical class determined by $A$ in $W$. Using this construction, Leavitt and Hoffmann have proved in [4] that if $A$ is a hereditary class (if $K \in A$ and $I$ is an ideal of $K$, then $I \in A$ ), then $L A$ is also hereditary. In this paper an alternate lower radical construction is given. As applications, a simple proof is given of the theorem of Leavitt and Hoffmann and a result of Yu-Lee Lee for alternative rings is extended to not necessarily associative rings.

Let $A \subseteq W$ be any class of rings. Define $R_{1}(A)$ to be the homomorphic closure of $A$. Proceeding inductively, let $\beta$ be an ordinal exceeding one and suppose the classes $R_{\alpha}(A)$ have been defined for all $\alpha<\beta$. If $\beta$ is not a limit ordinal, define

$$
R_{\beta}(A)=\left\{K \in W \mid I, K / I \in R_{\beta-1}(A) \text { for some } I<K\right\} .
$$

If $\beta$ is a limit ordinal, define

$$
\begin{gathered}
R_{\beta}(A)=\left\{K \in W \mid K \text { contains a chain }\left\{I_{\gamma}\right\}\right. \text { of ideals such that each } \\
\left.\qquad I_{\gamma} \in \bigcup_{\alpha<\beta} R_{\alpha}(A) \text {, and } K=\cup I_{\gamma}\right\} .
\end{gathered}
$$

Finally define $R(A)=\cup R_{\alpha}(A)$, where the union is taken over all ordinals $\alpha$.

The following characterization of radical classes is found in [2]. Using this characterization, we prove that $R(A)=L(A)$.

THEOREM 1. Let $W$ be a universal class and let $A \subseteq W$. Then $A$ is a radical class in $W$ if, and only if, the following conditions are satisfied:

i) $A$ is homomorphically closed

ii) If $I, K / I \in A$, then $K \in A$

iii) The union of a chain of $A$-ideals of a $W$-ring $K$ is again an $A$-ideal of $K$.

The following lemma is obvious.

LeMma 1. If $\alpha$ and $\beta$ are ordinals with $\alpha \leqq \beta$, then $R_{\alpha}(A) \subseteq R_{\beta}(A)$. 
Lemma 2. For every ordinal $\alpha \geqq 1, R_{\alpha}(A)$ is homomorphically closed. Hence $R(A)$ is homomorphically closed.

Proof. $R_{1}(A)$ is homomorphically closed. Let $\beta>1$ be an ordinal, and suppose $R_{\alpha}(A)$ is homomorphically closed for all $\alpha<\beta$. Let $K \in R_{\beta}(A)$ and let $I<K$. If $\beta$ is a limit ordinal, there is a chain $\left\{I_{\gamma}\right\}$ of ideals of $K$ such that $I_{\gamma}$ belongs to one of the classes $R_{\alpha}(A)$ with $\alpha<\beta$ and such that $K=\cup I_{\gamma}$. But $\left\{\left(I+I_{\gamma}\right) / I\right\}$ is a chain of ideals of $K / I$, and $K / I$ is its union. Since

$$
(I+I \gamma) / I \cong I \gamma /\left(I \cap I_{\gamma}\right),
$$

each of these ideals is a homomorphism of some $I_{\gamma}$, and thus by the induction hypothesis each $(I+I \gamma) / I$ belongs to some $R_{\alpha}(A)$ with $\alpha<\beta$. This means $K / I \in R_{\beta}(A)$.

Now suppose $\beta-1$ exists. Then $K$ contains an ideal $J$ so that $J, K / J \in R_{\beta-1}(A)$. By the induction hypothesis, $(J+I) / I$ and $K /(I+J)$ both belong to $R_{\beta-1}(A)$, since the former is a homomorphic image of $J$ and the latter of $K / J$. Since

$$
[R / I] /[(J+I) / I] \cong R /(J+I),
$$

$R / I \in R_{\beta}(A)$. Thus by transfinite induction $R_{\beta}(A)$ is homomorphically closed for all ordinals $\beta$. It follows immediately that $R(A)$ is homomorphically closed.

We now show that $R(A)$ satisfies conditions (ii) and (iii) of Theorem 1 .

Lemma 3. Let $K \in W$ and let $\left\{I_{\alpha}\right\}$ be a chain of $R(A)$-ideals of $K$. Then $\cup I_{\alpha}$ is an $R(A)$-ideal of $K$.

PROOF. Since $K$ is a set, there is by Lemma 1 an ordinal $\beta$ with the property that $I_{\alpha} \in R_{\beta}(A)$ for each $\alpha$. Let $\delta$ be a limit ordinal exceeding $\beta$, then $\cup I_{\alpha} \in R_{\delta}(A)$.

Lemma 4. Let $K \in W$, and suppose $K$ contains an ideal $I \in R(A)$ such that $K / I \in R(A)$. Then $K \in R(A)$.

Proof. By Lemma 1 , there is an ordinal $\beta$ such that $I, K / I \in R_{\beta}(A)$. This means that $K \in R_{\beta+1}(A)$.

THEOREM 2. $R(A)=L(A)$.

Proof. By Theorem 1 and Lemmas 2, 3, and 4, $R(A)$ is a radical class in $W$. By the minimality of $L(A)$ among radical classes in $W$ which contain $A$, it is enough to show $R(A) \subseteq L(A)$. This is accomplished by proving $R_{\alpha}(A) \subseteq L(A)$ for every ordinal $\alpha$.

Clearly $R_{1}(A) \subseteq L(A)$. Let $\beta$ be an ordinal exceeding one, and assume $R_{\alpha}(A) \subseteq L(A)$ for all ordinals $\alpha<\beta$. Let $K \in R_{\beta}(A)$. If $\beta$ is a limit ordinal, $K$ is the union of a chain of ideals from the classes $R_{\alpha}(A)$, where $\alpha<\beta$. Thus by the induction hypothesis $K$ is the union of $L(A)$-ideals, so $K \in L(A)$ by Theorem 1 . 
If $\beta$ is not a limit ordinal, there is an ideal $I$ of $K$ such that $I$ and $K / I$ both belong to $R_{\beta-1}(A) \subseteq L(A)$. Again, $K \in L(A)$ by Theorem 1 . Thus $R_{\beta}(A) \subseteq L(A)$ for all ordinals $\beta \geqq 1$.

The referee has provided an alternate proof that $L(A) \subseteq R(A)$, independent of Lemma 2 as follows.

Let $A_{\alpha}$ be the Kurosh classes (see [1]), then $A_{1}=R_{1}(A) \subseteq R(A)$. Let $\beta$ be an ordinal and suppose $A_{\alpha} \subseteq R(A)$ for all $\alpha<\beta$. Let $K \in A_{\beta}$ and let $S$ be the set of all $R(A)$-ideals of $K$. By Lemma $3 S$, is closed under taking unions of chains, so by Zorn's Lemma $S$ contains a maximal element $I$. If $I=K$ we are done, but if $0 \neq K / I$ there exists

$$
0 \neq J / 1<K / I \text { with } J / I \in A_{\alpha} \subseteq R(A) .
$$

By Lemma 4 we have $J \in R(A)$ contradicting the maximality of $I$. Hence $I=K \in R(A)$ so $A_{\alpha} \subseteq R(A)$ for each ordinal $\alpha$. Therefore $L A=\cup A_{\alpha} \subseteq R(A)$.

We now give a simple proof of the following theorem which appears in [4]. Other results of the form " $A$ has property $P$ implies $L A$ has property $P$ " may, perhaps, be provable in a similar way.

THEOREM 3. [4] Let $A \subseteq W$ where $W$ is some universal class. Then if $A$ is hereditary, so is $L(A)$.

Proof. We prove that $R_{\beta}(A)$ is hereditary for each $\beta \geqq 1$. This is easily seen to be true if $\beta=1$. Thus, assume $\beta>1$, and suppose $R_{\alpha}(A)$ is a hereditary class for each $\alpha<\beta$. Let $K \in R_{\beta}(A)$, and suppose $I$ is an ideal of $K$. If $\beta$ is a limit ordinal, $K=\cup I_{y}$ where $\left\{I_{y}\right\}$ is a chain of ideals each belonging to one of the (hereditary) classes $R_{\alpha}(A), \alpha<\beta$. But then $I=\cup\left(I_{y} \cap I\right)$ so $I \in R_{\beta}(A)$.

If $\beta$ is not a limit ordinal, there is an ideal $J$ of $K$ so that $J, K / J \in R_{\beta-1}(A)$. Since $R_{\beta-1}(A)$ is hereditary, $I \cap J$ and

$$
(J+I) / J \cong I /(I \cap J)
$$

both belong to $R_{\beta-1}(A)$. This implies $I \in R_{\beta}(A)$.

The proof of Theorem 4 requires the following lemma.

Lemma 5. If $P$ is a radical class in $W$ and for some $K^{\prime} \in W$ a subring $K \subseteq K^{\prime}$ is the set-theoretic union of $P$-ideals of $K^{\prime}$, then $K \in P$.

Proof. If $K=\cup I_{\alpha} \notin P$, then $K / I \in S P=\{H \in W \mid H$ has no nonzero $P$-ideals $\}$ for some $I \neq K$. Then for some $\alpha$ we have $I_{\alpha} \ddagger I$, so $\left(I_{\alpha}+I\right) I \cong$ $I_{\alpha} /\left(I \cap I_{\alpha}\right)$ is a nonzero $P$-ideal of $K / I$. This contradiction proves that $K \in P$.

The following theorem is proved for alternative rings in [7] by Yu-Lee Lee. 
THEOREM 4. If $A_{1}$ and $A_{2}$ are homomorphically closed, hereditary classes of W-rings, then $L\left(A_{1} \cap A_{2}\right)=L A \cap L A_{2}$.

Proof. Trivially $L\left(A_{1} \cap A_{2}\right) \subseteq L A_{1} \cap L A_{2}$. Since $K \in L A_{1} \cap L A_{2}$ if and only if $K \in R_{\gamma}\left(A_{1}\right) \cap R_{\gamma}\left(A_{2}\right)$ for some ordinal number $\gamma$. It suffices to prove

$$
\left.R_{\gamma}\left(A_{1}\right) \cap R_{\gamma}\left(A_{2}\right) \subseteq L A_{1} \cap A_{2}\right),
$$

for each ordinal $\gamma \geqq 1$. This is clear for $\gamma=1$. Let $\beta$ be an ordinal number greater than 1 and suppose

$$
R_{\alpha}\left(A_{1}\right) \cap R_{\alpha}\left(A_{2}\right) \subseteq L\left(A_{1} \cap A_{2}\right)
$$

for each ordinal $\alpha<\beta$. Let $K \in R_{\beta}\left(A_{1}\right) \cap R_{\beta}\left(A_{2}\right)$.

If $\beta$ is a limit ordinal, $K$ is the union of a chain $\left\{I_{\gamma}\right\}_{\gamma \in C}$ of ideals each belonging to one of the classes $R_{\alpha}\left(A_{1}\right)$ for $\alpha<\beta$. Also $K$ is the union of a chain $\left\{J_{\delta}\right\}_{\delta \in D}$ of ideals each belonging to one of the classes $R_{\alpha}\left(A_{2}\right)$ for $\alpha<\beta$. If $x \in K, x \in J \delta$ for some $\delta \in D$ and $x \in I_{\gamma}$ for some $\gamma \in C$, so $x \in J_{\delta} \cap I_{\gamma}$ for some $(\delta, \gamma) \in D \times C$. Since $J_{\delta} \in R_{\alpha}\left(A_{2}\right)$ for some $\alpha<\beta$, and since $R_{\alpha}\left(A_{2}\right)$ is hereditary (see proof of Theorem 3), $J_{\delta} \cap I_{\lambda} \in R_{\alpha}\left(A_{2}\right)$. Similarly $J_{\delta} \cap I_{\gamma} \in R_{\eta}\left(A_{1}\right)$ for some $\eta<\beta$. Thus

$$
J_{\delta} \cap I_{\gamma} \in R_{\mu}\left(A_{1}\right) \cap R_{\mu}\left(A_{2}\right),
$$

where $\mu=\max [\eta, \alpha]$. Since $\mu<\beta$, the induction hypothesis implies $J_{\delta} \cap I_{y} \in L\left(A_{1} \cap A_{2}\right)$ so that $K$ is the set-theoretic union of $L\left(A_{1} \cap A_{2}\right)$-ideals. Thus, by Lemma $5, K \in L\left(A_{1} \cap A_{2}\right)$.

Now suppose $\beta-1$ exists, and let $K \in R_{\beta}\left(A_{1}\right) \cap R_{\beta}\left(A_{2}\right)$. Then there exist ideals $I$ and $J$ such that $I, K / I \in R_{\beta-1}\left(A_{1}\right)$ and $J, K / J \in R_{\beta-1}\left(A_{2}\right)$. Since $R_{\beta-1}\left(A_{1}\right)$ and $R_{\beta-1}\left(A_{2}\right)$ are hereditary,

$$
I \cap J \in R_{\beta-1}\left(A_{1}\right) \cap R_{\beta-1}\left(A_{2}\right)
$$

so $I \cap J \in L\left(A_{1} \cap A_{2}\right)$. Since $R_{\beta-1}\left(A_{1}\right)$ is homomorphically closed (Lemma 2),

$$
I /(I \cap J) \cong(I+J) / J \in R_{\beta-1}\left(A_{1}\right) .
$$

Since $R_{\beta-1}\left(A_{2}\right)$ is hereditary, $(I+J) / J$, as an ideal of $K / J$ is a member of $R_{\beta-1}\left(A_{2}\right)$. Thus

$$
I /(I \cap J) \cong(I+J) / J \in R_{\beta-1}\left(A_{1}\right) \cap R_{\beta-1}\left(A_{2}\right) \subseteq L\left(A_{1} \cap A_{2}\right) .
$$

Thus $I \cap J$ and $I /(I \cap J)$ belong to $R_{\beta-1}\left(A_{1}\right) \cap R_{\beta-1}\left(A_{2}\right) \subseteq L\left(A_{1} \cap A_{2}\right)$.

Thus since $I \cap J$ and $I /(I \cap J)$ belong to $L\left(A_{1} \cap A_{2}\right), I \in L\left(A_{1} \cap A_{2}\right)$. Similarly, $J \in L\left(A_{1} \cap A_{2}\right)$ so that $I+J$ is an $L\left(A_{1} \cap A_{2}\right)$-ideal of $K$. Also $K /(I+J)$ belongs to

$$
R_{\beta-1}\left(A_{1}\right) \cap R_{\beta-1}\left(A_{2}\right) \subseteq L\left(A_{1} \cap A_{2}\right)
$$


since it is the homomorphic image of both $K / J$ and $K / I$. Thus, since $I+J$ and $K /(I+J)$ belong to $L\left(A_{1} \cap A_{2}\right)$, we have that $K \in L\left(A_{1} \cap A_{2}\right)$.

We have shown that $R_{\beta}\left(A_{1}\right) \cap R\left(A_{2}\right) \subseteq L\left(A_{1} \cap A_{2}\right)$ which proves the theorem.

COROLLARY. If $A_{i}, i=1,2, \cdots, n$, are homomorphically closed, hereditary classes of W-rings, then $L\left(\bigcap_{i=1}^{n} A_{i}\right)=\bigcap_{i=1}^{n} L A_{i}$.

Proof. By induction.

It is shown in [1] that the Kurosh-Amitsur construction terminates at $\omega$, the first infinite ordinal in case $W$ is an associative universal class. If $A$ is hereditary in an associative class, then $L A=A_{3}$, the third step in the KuroshAmitsur construction (see [3]). To see that similar properties do not hold for the construction of Theorem 2 , let $W$ be the class of all associative rings and $Z \subseteq W$ the class of rings having zero multiplication. Then the classes $R_{n}(Z), n$ finite, are all distinct. Jacobson has given in [5] an example of an $L Z$-ring $K$ which is not the sum (and thus not the union) of its nilpotent ideals. Therefore $K \notin R_{\omega}(Z)$ so that $L Z \neq R_{\omega}(Z)$.

\section{References}

[1] T. Anderson, N. Divinsky, and A. Sulinsky, 'Lower, Radical Properties for Associative and Alternative Rings'; J. London Math. Soc. 41 (1966), 417-424.

[2] S. A. Amitsur, 'Radicals in Rings and Bicategories', Amer. J. Math. 76 (1954), 100-125.

[3] E. P. Armendariz and W. G. Leavitt, 'The Hereditary Property in the Lower Radical Construction', Canad. J. Math. 20 (1968), 474-476.

[4] A. E. Hoffman and W. G. Leavitt, 'Properties Inherited by the Lower Radical.' Protugaliae Mathematica 27 (1968), 63-66.

[5] N. Jacobson, Structure of Rings (Amer. Math. Soc. Coll. Publ. 37, Providence, 1956).

[6] A. Kurosh, 'Radicals in Rings and Algebras', Math. Sb. 33, (1953), 13-26.

[7] Yu-Lee Lee, 'On Intersections and Unions of Radical Classes', J. Aust. Math. Soc. (To appear).

University of Florida

Western Illinois University

Macomb, Illinois 61455

U.S.A. 